OPEN ACCESS

Edited by:

Chunjie Jiang,

University of Pennsylvania,

United States

Reviewed by:

Yingjie Zhang,

Wistar Institute, United States

Shunmin $\mathrm{He}$,

Institute of Biophysics (CAS), China

Chong Jin,

University of Pennsylvania,

United States

*Correspondence:

Fei Su

sufei4818@bjhmoh.cn

Yaqun Zhang

zhangyaqun3365@bjhmoh.cn

${ }^{\dagger}$ These authors have contributed

equally to this work

Specialty section:

This article was submitted to

Computational Genomics,

a section of the journal

Frontiers in Genetics

Received: 01 December 2020

Accepted: 06 January 2021

Published: 26 January 2021

Citation:

Zhang L, Zhang W, Li H, Tang $X$,

Xu S, Wu M, Wan L, Su F and

Zhang Y (2021) Five Circular RNAs

in Metabolism Pathways Related

to Prostate Cancer.

Front. Genet. 12:636419.

doi: 10.3389/fgene.2021.636419

\section{Five Circular RNAs in Metabolism Pathways Related to Prostate Cancer}

\author{
Lili Zhang't, Wei Zhang't, Hexin Li', Xiaokun Tang', Siyuan Xu', Meng Wü, Li Wan', \\ $\mathrm{Fei} \mathrm{Su}^{1 *}$ and Yaqun Zhang ${ }^{3 *}$ \\ ${ }^{1}$ Clinical Biobank, Beijing Hospital, National Center of Gerontology, National Health Commission, Institute of Geriatric \\ Medicine, Chinese Academy of Medical Sciences, Beijing, China, ${ }^{2}$ Department of Pathology, Beijing Hospital, National \\ Center of Gerontology, National Health Commission, Institute of Geriatric Medicine, Chinese Academy of Medical Sciences, \\ Beijing, China, ${ }^{3}$ Department of Urology, Beijing Hospital, National Center of Gerontology, National Health Commission, \\ Institute of Geriatric Medicine, Chinese Academy of Medical Sciences, Beijing, China, ${ }^{4}$ The Key Laboratory of Geriatrics, \\ Beijing Institute of Geriatrics, Beijing Hospital, National Center of Gerontology, National Health Commission, Institute of \\ Geriatric Medicine, Chinese Academy of Medical Sciences, Beijing, China
}

Prostate cancer ( $\mathrm{PCa}$ ) is the most common malignant tumor in men, and its incidence increases with age. Serum prostate-specific antigen and tissue biopsy remain the standard for diagnosis of suspected PCa. However, these clinical indicators may lead to aggressive overtreatment in patients who have been treated sufficiently with active surveillance. Circular RNAs (circRNAs) have been recently recognized as a new type of regulatory RNA that is not easily degraded by RNases and other exonucleases because of their covalent closed cyclic structure. Thus, we utilized high-throughput sequencing data and bioinformatics analysis to identify specifically expressed circRNAs in PCa and filtered out five specific circRNAs for further analysishsa_circ_0006410, hsa_circ_0003970, hsa_circ_0006754, hsa_circ_0005848, and a novel circRNA, hsa_circ_AKAP7. We constructed a circRNA-miRNA regulatory network and used miRNA and differentially expressed mRNA interactions to predict the function of the selected circRNAs. Furthermore, survival analysis of their cognate genes and PCR verification of these five circRNAs revealed that they are closely related to well-known PCa pathways such as the MAPK signaling pathway, P53 pathway, androgen receptor signaling pathway, cell cycle, hormone-mediated signaling pathway, and cellular lipid metabolic process. By understanding the related metabolism of circRNAs, these circRNAs could act as metabolic biomarkers, and monitoring their levels could help diagnose PCa. Meanwhile, the exact regulatory mechanism for AR-related regulation in $\mathrm{PCa}$ is still unclear. The circRNAs we found can provide new solutions for research in this field.

Keywords: prostate cancer, circular RNA, miRNA-mRNA, pathways, bioinformatics

\section{INTRODUCTION}

Prostate cancer (PCa) is a slow-growing malignant tumor, the incidence of which increases with age (Carroll and Mohler, 2018; Etzioni and Nyame, 2020). At the beginning of diagnosis, most patients are asymptomatic; however, it is still among the top three causes of cancer-related deaths in men (Siegel et al., 2017). Patients with a high risk of PCa must undergo periodic testing for serum prostate-specific antigen (PSA). Tissue biopsy remains the care standard for diagnosis for 
suspected PCa (Litwin and Tan, 2017). After the tumor is confirmed by biopsy, the next step is to determine the invasiveness of tumor cells. The Gleason score is the most commonly used scale to assess the grade of $\mathrm{PCa}$. When the grade is high, tumors tend to spread. Most Gleason scores used to evaluate prostate biopsy samples range from 6 to 10. A score of 6 indicates low-risk PCa; a score of 7 indicates intermediate-risk PCa; a score of 8 to 10 indicates high-risk PCa (2019). However, researchers have found that the "normal" PSA level of 0-4 ng/mL does not guarantee cancer-free status; in approximately $25 \%$ of men with a PSA below $4 \mathrm{ng} / \mathrm{mL}$, a biopsy still reveals PCa (Kitagawa et al., 2014). Thus, these clinical indexes cannot guarantee the reliability of diagnosis. New assistant biomarkers need to be developed for the diagnosis of PCa.

With the development of sequencing technology, circRNAs are recognized as a new type of regulatory RNA. They were first identified by analysis using next generation RNA sequencing (RNA-seq) in a study of pediatric acute lymphoblastic leukemia (Salzman et al., 2012). Most circRNAs are composed of protein-coding exons; thus, the expression of these circRNAs competes with the production of pre-mRNAs. These events also lead to the expression of circRNAs being higher than that of their cognate linear RNAs under certain conditions (Jeck et al., 2013; Jeck and Sharpless, 2014). CircRNAs can function by directly regulating gene expression or by acting as miRNA sponges (Tay et al., 2014). They are similar to competitive endogenous RNAs (ceRNAs) and contain miRNA response elements (MREs). Therefore, they can function by competing with mRNAs to bind miRNAs (Hansen et al., 2013). For example, dysregulation of circRNA-0001946 contributes to tumor cell proliferation and metastasis in colorectal cancer by targeting microRNA-135a-5p (Deng et al., 2020). CircRNAs are not easily degraded by RNases and other exonucleases due to a covalent closed cyclic structure without free $5^{\prime}$ or $3^{\prime}$ ends. They have a longer half-life $(>48 \mathrm{~h})$ than linear RNAs (Suzuki et al., 2006; Jeck and Sharpless, 2014). CircRNAs have been known to be rich in tumors (Salzman et al., 2012). Therefore, compared with other RNAs, circRNAs have more advantages as novel biomarkers of cancer and other diseases (Arnaiz et al., 2019).

Studies have shown that circRNAs are functional in PCa. Overexpression of circ0005276 and its host gene X-linked inhibitor of apoptosis protein (XIAP) can promote cell proliferation, migration, and epithelial-mesenchymal transition in PCa tissues compared with that in normal tissues (Feng et al., 2019). CircRNAs can act as oncogenes in the progression of PCa and are differentially expressed between cancer tissues and normal tissues (Feng et al., 2019). It is also reported that circRNAs can act as therapeutic targets. For example, the overexpression of circRNA cir-ITCH significantly inhibits the proliferation, migration, and invasion of $\mathrm{PCa}$ cells. By targeting miR-17 in PC-3 and LNCaP cell lines, circRNAs could act as therapeutic targets in PCa, especially in castration-resistant prostate cancer (CRPC) (Li et al., 2020). CircRNAs also affect carbohydrate, lipid, and amino acid metabolism in cancer. By regulating transcription factors, circRNAs can modulate glycolysis (Yu et al., 2019). Thus, it is important to identify differentially expressed circRNAs in $\mathrm{PCa}$ and explore their potential as diagnostic and therapeutic targets in cancer.

In this study, we compared circRNAs between four PCa tissues and two adjacent normal tissues of two PCa patients by sequencing six sets of RNA-seq. We selected five circRNAs that were highly expressed in tumor tissues and found that the fold change in expression of these five circRNAs was significantly higher than that of their cognate linear RNAs. We verified these circRNAs by PCR in PCa cell lines and used the circRNAmiRNA-mRNA method to predict biological pathways regulated by these circRNAs. Some well-known pathways in PCa were enriched, such as the p53 signaling pathway, MAPK signaling pathway, hormone-mediated signaling pathway, and cellular lipid metabolic process. These pathways also confirmed the high reliability of the five circRNAs that participated in the regulation of $\mathrm{PCa}$.

\section{MATERIALS AND METHODS}

\section{Patients and Samples}

For sequencing samples, two pairs of PCa tissues and adjacent tissues were derived from surgical samples. Sections from normal and malignant tissues were examined after staining with hematoxylin and eosin. The tumor specimen comprised $>80 \%$ malignant cells, and the benign specimen comprised an approximately equal admixture of normal epithelial and stromal cells. The pathology of the prostate tumor was checked by a pathologist and established as a combined Gleason Score of $6(3+3)$, stage $\mathrm{T} 2 \mathrm{a}$, with focal involvement of the surgical margin. RNA was purified from minced frozen tissue using Trizol reagent (Life Technologies, Inc., Rockville, MD, United States). Total RNA was briefly treated with DNase I. For each sample, an RNA library was constructed using $3 \mu \mathrm{g}$ total RNA. Ribo-Zero Gold Kits were used to remove rRNA. According to the instructions of the NEB-Next Ultra Directional RNA Library Prep Kit for Illumina (NEB, Ispawich, United States), different index tags were selected. The constructed libraries were sequenced using Illumina; the sequencing strategy used was PE150.

For qRT-PCR, 20 pairs of PCa tissues and adjacent normal tissues were collected from the Department of Pathology of Beijing Hospital with Gleason scores of 6 (14 cases) and 7 (6 cases). All tissues were fixed in phosphate-buffered formalin, dehydrated with ethanol, and embedded in paraffin. The malignant status and Gleason score were obtained for these samples by histological analysis. The work was approved by the Beijing Hospital Ethics Committee.

None of these patients had undergone hormonal therapy prior to surgery.

\section{Quality Control and Mapping of Sequencing Data}

The quality of the fastq data of RNA-seq was evaluated using fastQC (Andrews, 2010). We found some reads mixed with adapters, and then Trimmomatic (Bolger et al., 2014) was used 
to filter these sequences. The reads with lengths of less than 28 were dropped. The read quality was filtered through a four-base sliding window with an average quality threshold of 15 . After the reads passed the sequence quality tests, the filtered reads were mapped to the human hg38 genome (Lander et al., 2001) using the aligner software STAR (Dobin et al., 2013) with parameter “-chimSegmentMin 10."

\section{Differential Expressed circRNAs Filtered}

CIRCexplorer2 (Zhang et al., 2016) and CLEAR/CIRCexplorer3 (Ma et al., 2019) were mainly used to obtain circRNAs in our research. For CIRCexplorer2, we used the parse module to analyze circRNA fusion junction reads and annotate modules for circRNA gene information. The expression of circRNAs was quantified by CIRCscore in CLEAR/CIRCexplorer3, which indicates the circRNA expression level by linear RNA expression level adjustment. The expression of fold change between tumor circRNAs and normal adjacent prostatic tissue circRNAs was calculated to filter tumor-specific expressed circRNAs.

\section{Bioinformatics Analysis of CircRNAs}

All of the interaction binding sites between circRNAs and miRNAs were downloaded from circBank (Liu et al., 2019). For the novel circRNAs, we used miRNDB (Chen and Wang, 2020) to predict the related miRNAs. We identified the function of the predicted miRNAs by manual literature mining. Then, Cytoscape (Shannon et al., 2003) was used to build a network between circRNAs and miRNAs. For differentially expressed mRNAs, we chose featureCounts (Liao et al., 2014) to quantify read counts for each gene. Based on paired-end data, "requireBothEndsMapped = TRUE" and "isPairedEnd = TRUE" were set additionally. Then, we calculated the normalized expression levels in fragments per kilobase per million mapped reads (FPKM) by using the DGEList and rpkm function from edgeR (Robinson et al., 2010).

To predict circRNA-related pathways, we regarded miRNAs as a middleman to find circRNA-related mRNAs. The interactions between miRNAs and mRNAs were obtained from miRDB (Chen and Wang, 2020). Meanwhile, it provided interaction scores to assess accuracy; only scores higher than 90 and differentially expressed mRNAs were considered for further analysis.

\section{Functional Enrichment Analysis and Survival Analysis}

The circRNA-related mRNA list was analyzed using the functional enrichment tool GOseq (Young et al., 2010). Compared with other tools, GOseq can alleviate selection bias more effectively. The pathways were drawn using ggplot2 (Wickham, 2016) in the R language. For survival analysis, TCGA PCa (PRAD) data were selected in UCSC Xena (Goldman et al., 2020) with progression free intervals to draw Kaplan-Meier plots of circRNA cognate genes. The expression level of the gene was used for survival analysis.

\section{RNA Extraction and Real-Time Quantitative PCR (qPCR)}

A total of $20 \mathrm{PCa}$ tissues and 20 adjacent normal tissue samples were prepared. RNA was extracted from three 10$\mu \mathrm{m}$ FPE sections per sample. Paraffin was removed by xylene extraction followed by washing with ethanol. RNA was isolated from the sectioned tissue blocks using the purification kit, total RNA was extracted, and RNA was subjected to DNase I (Invitrogen, AM2222) treatment. The qRT-PCR was performed using the TransScript II Green One-Step qRT-PCR SuperMix kit (TransGen Biotech, AQ311-01) with 100 ng RNA as template in a $20 \mu \mathrm{L}$ reaction volume on an ABI 7,500 real-time cycler (Qiagen).

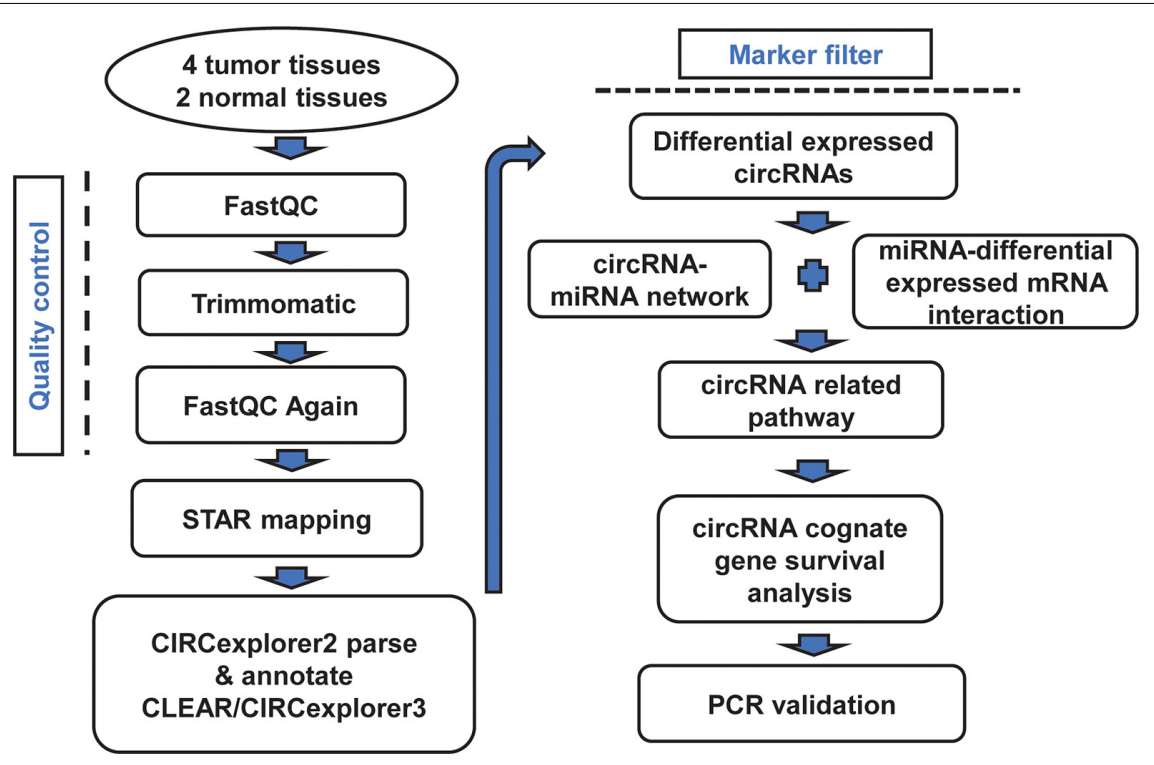

FIGURE 1 | Bioinformatics analysis pipeline of our study. 
Fold change of circRNA in PCa vs. adjacent normal tissue

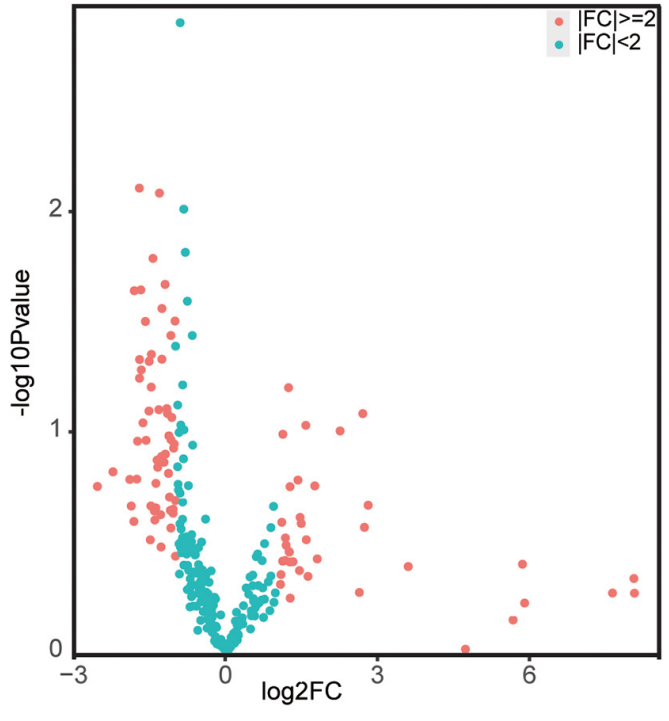

B

C
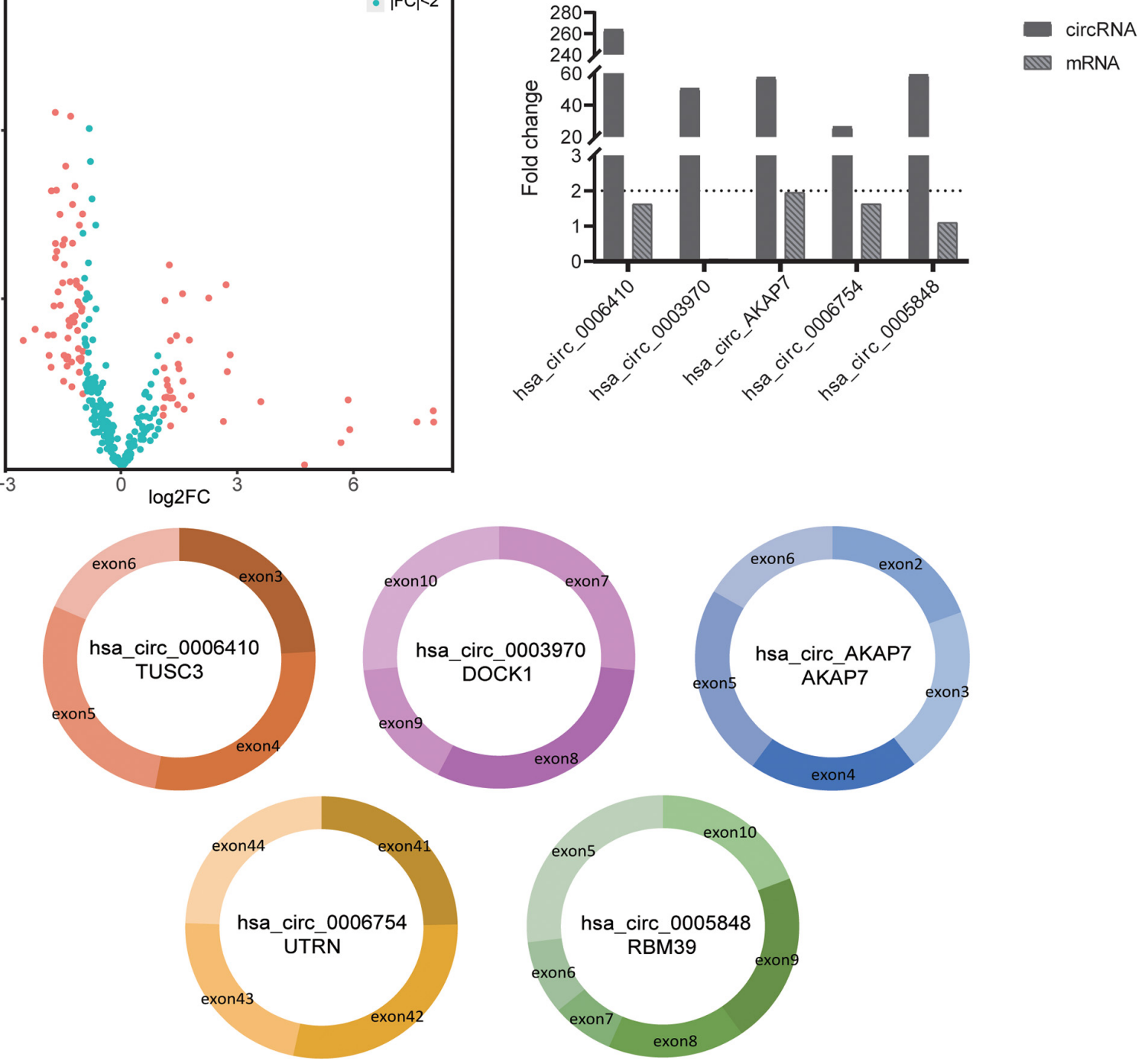

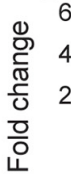

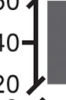

$\ldots \ldots . . . \cdots \cdots$

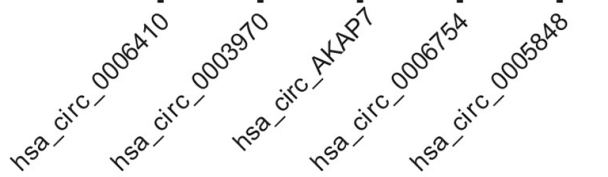

D
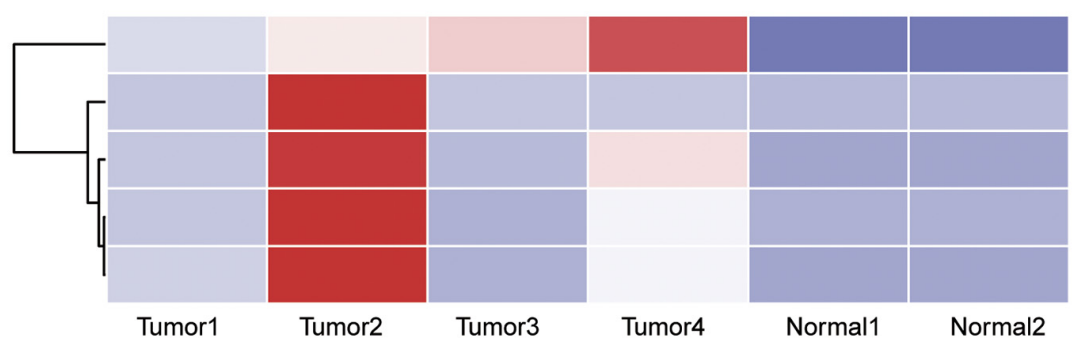

hsa_circ_0006754

hsa_circ_0006410

hsa_circ_0003970

hsa_circ_AKAP7

hsa_circ_0005848

Normal1

Normal2

FIGURE 2 | Information of the five PCa specific circRNAs. (A) Volcano plot of circRNAs in PCa. The red points represent differentially expressed circRNAs in PCa with fold-change $>2$ or fold-change $<0.5$. The paired $t$-test was used to obtain $p$-value. (B) The fold-change of circRNAs and its cognate mRNA expression between tumor tissue and adjacent normal tissue. The fold-change of circRNAs was much higher than its mRNAs especially in hsa_circ_0006410. In both tumor as well as normal samples, the expression of hsa_circ_0003970 cognate mRNA was 0. Thus, the mRNA fold change of hsa_circ_0003970 was 0. (C) The exon composition of hsa_circ_0006410, hsa_circ_0003970, hsa_circ_0006754, hsa_circ_0005848, and hsa_circ_AKAP7. (D) Heatmap of the expression of five specific circRNAs. These five circRNAs are highly expressed in tumor tissues than in normal tissues. The value used in this figure is the expression of circRNAs, and we used "scale = row" to make the graph more consecutive. 
TABLE 1 | The detailed information of circRNAs.

\begin{tabular}{|c|c|c|c|c|c|c|c|}
\hline circRNA ID & Chrom & Start & End & Strand & Cognate mRNA & Cognate gene & Pearson correlation \\
\hline hsa_circ_0006410 & chr8 & 15650696 & 15673836 & + & ENST00000382020.8 & TUSC3 & 0.997861 \\
\hline hsa_circ_0003970 & $\operatorname{chr10}$ & 126996747 & 127000307 & + & ENST00000280333.9 & DOCK1 & 0.962136 \\
\hline hsa_circ_AKAP7 & chr6 & 131145284 & 131199573 & + & ENST00000431975.7 & AKAP7 & -0.31984 \\
\hline hsa_circ_0006754 & chr6 & 144531051 & 144539443 & + & ENST00000367545.7 & UTRN & 0.841363 \\
\hline hsa_circ_0005848 & chr20 & 35721739 & 35732135 & - & ENST00000639702.1 & RBM39 & 0.97713 \\
\hline
\end{tabular}

The genome version is hg38.

PCR cycling was performed as follows: one cycle at $95^{\circ} \mathrm{C}$ for $10 \mathrm{~min}, 95^{\circ} \mathrm{C}$ for $20 \mathrm{~s}$, and 40 cycles at $60^{\circ} \mathrm{C}$ for $45 \mathrm{~s}$. The threshold cycle for a given amplification curve during RT-PCR occurs at the point where the fluorescent signal grows beyond a specified fluorescence threshold setting.

The results were normalized with beta actin, and the relative RNA expression was calculated by the 2$\Delta \Delta \mathrm{Ct}$ method. To evaluate the statistical significance of PCR data, the paired sample $t$-test was used. The hsa_circ_0003970 primer sequences were as follows: left primer 5'-AGCTGAGGGACAACAACACC-3'; right primer 5'-CCTCTTGTAACCTTTCCTCCA-3'. The hsa_circ_0006410 primer sequences were as follows: left primer $5^{\prime}$-GTGGA ACCATATCCGTGGAC-3'; right primer 5'-GAAAAACGTCT GTCCCCTCA-3'. The hsa_circ_0006754 primer sequences were as follows: left primer $5^{\prime}$-CTGAATTGGAGATGCTTTCAGA3'; right primer 5'-TGGAGCACAGGTATCAACCA-3'. The hsa_circ_0005848 primer sequences were as follows: left primer 5'-GGGAAGTGCTGGACCTATGA-3'; right primer $5^{\prime}$-TCACGGCTTTTGCTCTTTTT-3'. The hsa_circ_AKAP7 primer sequences were as follows: left primer $5^{\prime}$-AGGCA TCCTGGTAGGAGAGAG-3'; right primer $5^{\prime}$-AGCAAATGG CATGTCTACCA-3'.

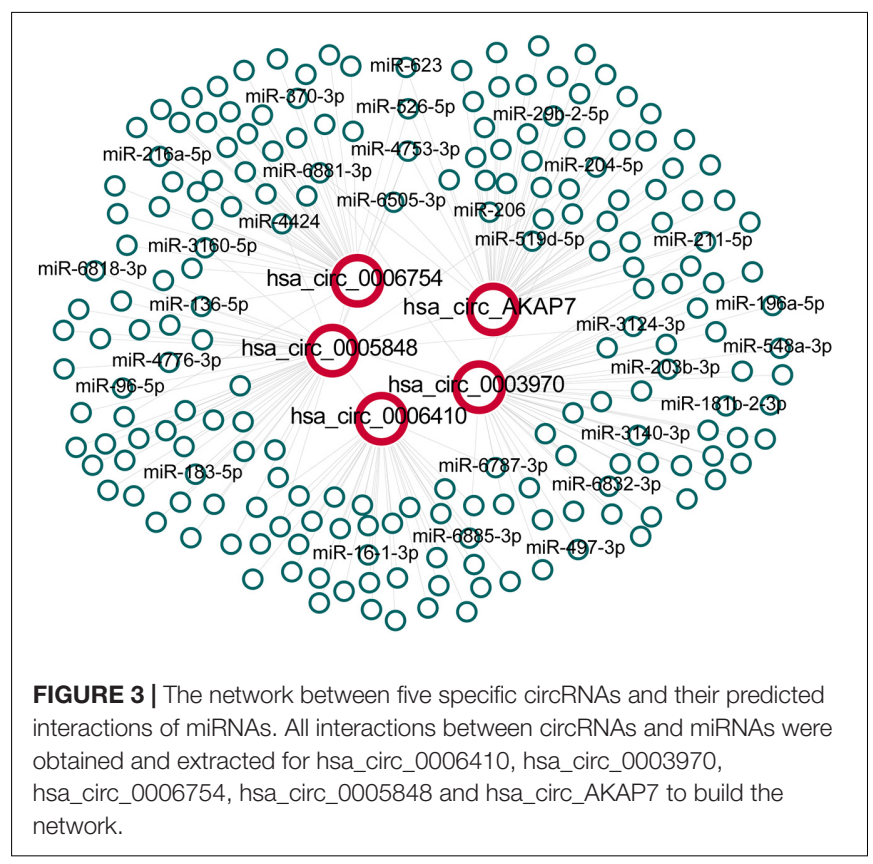

\section{RESULTS}

\section{Differentially Expressed CircRNAs in PCa}

Analysis of RNA-seq data (Figure 1) showed 89 differentially expressed circRNAs with fold-change $>2$ or fold-change $<0.5$. Due to the small sample size in our sequencing control set, the $p$-value was not accurate for initial screening. The differentially expressed circRNAs included 32 upregulated circRNAs and 57 downregulated circRNAs (Figure 2A). According to this result, we selected the top five differentially expressed circRNAs for further analysis_included hsa_circ_0006410, hsa_circ_0003970, hsa_circ_0006754, hsa_circ_0005848, and a novel circRNA that we named hsa_circ_AKAP7 (Figure 2C and Table 1). Because of the circRNA back-splicing feature, an mRNA may correspond to multiple circRNAs. Figure 2C shows the precise corresponding exon, enabling the identification of its component. These five circRNAs were highly expressed in tumor tissues compared with their cognate mRNAs (Figure 2B). The Pearson correlation coefficients of circRNAs and mRNAs were consistent with those found in previous studies; circRNAs and mRNAs tend to have a high correlation (Yang et al., 2018). The produced circRNAs and their cognate mRNAs usually inhibit each other but Figure 2B did not show an opposite trend of circRNA and mRNA expression. This indicated that these five circRNAs have key functions in tumor tissue instead of their cognate mRNAs. The expression of these five circRNAs is depicted in Figure 2D. The prominent higher expression of these five specific circRNAs led us to further research. Therefore, we regarded circRNAs as miRNA sponges to explore the functions of these five circRNAs.

\section{CircRNA-miRNA Network and miRNA-Related mRNAs}

After obtaining circRNA and miRNA interactions from circBank and miRNDB, we built five circRNA and miRNA interaction networks (Figure 3). We found 215 circRNAmiRNA interactions in the network, and each circRNA had an average of 43 miRNA interactions. Many of these miRNAs have been reported to be associated with PCa (Table 2). Hsa_circ_0003970 and hsa_circ_0005848 interacted with miRNA-204-5p, which is a tumor suppressor that promotes apoptosis by targeting BCL2 in PCa cells (Lin et al., 2017). Hsa_circ_0005848 and hsa_circ_0006754 related miR$3160-5 \mathrm{p}$ is a PCa cell proliferation suppressor that targets the F-box protein (Lin et al., 2018). MiR-548 acts as an 
anti-oncogenic factor that inhibits the phosphoinositide three-kinase (PI3K)/AKT signaling pathway in lung cancer and is associated with high-risk Gleason scores in PCa (Shi et al., 2015). The PI3K/AKT pathway is involved in tumor immunological surveillance and immune suppression (Dituri et al., 2011). Hsa-miR-181b-2-3p and hsa-miR-96-5p were associated with the androgen receptor and Gleason score (Mekhail et al., 2014). These results further highlight the contribution of this study.

The miRNA target prediction based on the short seed sequence provided many false positive results. Thus, we filtered differentially expressed mRNAs in PCa to analyze miRNA and mRNA interactions. Only scores $>90$ interactions were selected to predict circRNAs function. Then, we used functional enrichment analysis to explore the function of the five circRNAs.

\section{CircRNA-Related Pathways in Metabolism Pathways}

Analysis of these five circRNA-related mRNAs showed that they were all enriched in many well-known PCa pathways (Figure 4A). Hsa_circ_0006410, hsa_circ_0003970, hsa_circ_AKAP7, hsa_circ_0006754, and hsa_circ_0005848 were all related to the MAPK signaling pathway. MAPK signaling is an important regulator of cancer, especially PCa. It includes three cross-signaling pathways: p38, JNK, and ERK (Dhillon et al., 2007). Each pathway comprises several levels of kinases. The p38-MAPK pathway is important for the production of inflammatory cytokines and IFN- $\gamma$. It can also positively regulate Th1 differentiation instead of Th2 (Martinez et al., 2009). The JNK-MAPK pathway plays pro-inflammatory roles in macrophages, inducing M1 differentiation. Activation of the ERK-MAPK pathway favors cell differentiation into CD4 lineage and is critical for CD4 $\mathrm{T}$ cell polarization of $\mathrm{Th} 2$ because it is required for IL-4 receptor function (Alessandro et al., 2019). These regulators are significant in $\mathrm{PCa}$.

Other significant pathways were the hormone-mediated signaling pathway and cellular lipid-related process, which were associated with hsa_circ_0006410, hsa_circ_0003970, hsa_circ_AKAP7, hsa_circ_0006754, and hsa_circ_0005848 (Figures 4B-F). Steroid androgen hormones play key roles in the progression and treatment of PCa. Androgen deprivation therapy (ADT) is the first-line treatment used to control cancer growth (Munkley et al., 2016). It functions by inhibiting the production of male hormone testosterone and preventing it from reaching PCa cells. ADT can cause apoptosis of PCa cells and can make them grow slowly. Studies have indicated that dietary fat intake is related to $\mathrm{PCa}$ development, suggesting that lipid metabolism plays a role in the carcinogenesis and progression of PCa (Tamura et al., 2009). Dysregulation of metabolism of lipids, especially sphingolipid, is a hallmark of the malignant phenotype. Increased lipid accumulation leading to changes in levels of lipid metabolic enzymes has been verified in various tumors, including $\mathrm{PCa}$ (Wu et al., 2014). Castration-resistant PCa (CRPC) is considered to utilize de novo lipid synthesis to produce fatty acids to obtain energy (Eidelman et al., 2017). The five circRNAs were related to both the hormone-mediated signaling pathway and the lipid-related process, indicating that they are involved in $\mathrm{PCa}$ regulation. Several pathways found to be closely related to PCa include the chemokine pathway, cell cycle, p53 signaling

TABLE 2 | The literature mining of circRNAs related miRNAs.

\begin{tabular}{|c|c|c|c|}
\hline circRNA ID & miRNA ID & miRNA description in PCa or other tumors & References \\
\hline hsa_circ_0003970 & hsa-miR-181b-2-3p & AR signaling in PCa; cancer stem cell (CSC) formation in PCa & Mekhail et al., 2014 \\
\hline hsa_circ_0003970 & hsa-miR-196a-5p & Associated with SNPs that can be useful in screening for cancer risk & Mekhail et al., 2014 \\
\hline hsa_circ_0003970 & hsa-miR-203b-3p & Anti-metastatic in PCa; epithelial to mesenchymal transition (EMT) in PCa & Mekhail et al., 2014 \\
\hline hsa_circ_0003970 & hsa-miR-211-5p & Tumor suppressor by targeting ACSL4 in Hepatocellular Carcinoma & Qin et al., 2020 \\
\hline hsa_circ_0003970 & hsa-miR-497-3p & Down-regulated in $\mathrm{PCa}$ & Mekhail et al., 2014 \\
\hline hsa_circ_0003970 & hsa-miR-548a-3p & $\begin{array}{l}\text { Anti-oncogenic factor inhibiting the PI3K/AKT signaling pathway in lung cancer and } \\
\text { associated with high-risk Gleason scores in prostate cancer }\end{array}$ & Li et al., 2013 \\
\hline $\begin{array}{l}\text { hsa_circ_0003970; } \\
\text { hsa_circ_0005848 }\end{array}$ & hsa-miR-204-5p & $\begin{array}{l}\text { Tumor suppressor miRNA-204-5p promotes apoptosis by targeting } \\
\text { BCL2 in PCa }\end{array}$ & Mekhail et al., 2014 \\
\hline hsa_circ_0006754 & hsa-miR-216a-5p & $\begin{array}{l}\text { Inhibits malignant progression in small cell lung cancer: involvement of the Bcl-2 } \\
\text { family proteins }\end{array}$ & Sun et al., 2018 \\
\hline hsa_circ_0006754 & hsa-miR-370-3p & Up-regulated in PCa & Mekhail et al., 2014 \\
\hline $\begin{array}{l}\text { hsa_circ_0006754; } \\
\text { hsa_circ_AKAP7 }\end{array}$ & hsa-miR-526b-5p & $\begin{array}{l}\text { hsa_circ_0085539 promotes osteosarcoma progression by regulating miR-526b-5p } \\
\text { and SERP1 }\end{array}$ & Mekhail et al., 2014 \\
\hline hsa_circ_0006410 & hsa-miR-16-1-3p & Biochemical failure in $\mathrm{PCa}$ & Mekhail et al., 2014 \\
\hline hsa_circ_0005848 & hsa-miR-183-5p & Up-regulated in PCa & Mekhail et al., 2014 \\
\hline hsa_circ_0005848 & hsa-miR-3160-5p & Suppressed prostate cancer cell proliferation & Lin et al., 2018 \\
\hline hsa_circ_0005848 & hsa-miR-96-5p & Biochemical failure in PCa; Gleason score in $\mathrm{PCa}$ & Mekhail et al., 2014 \\
\hline $\begin{array}{l}\text { hsa_circ_0005848; } \\
\text { hsa_circ_AKAP7 }\end{array}$ & hsa-miR-623 & Suppressed tumor progression in human lung adenocarcinoma & Wei et al., 2016 \\
\hline has_circ_AKAP7 & hsa-miR-206 & Anti-metastatic in $\mathrm{PCa}$ & Mekhail et al., 2014 \\
\hline has_circ_AKAP7 & hsa-miR-29b-2-5p & Anti-metastatic in $\mathrm{PCa}$ & Mekhail et al., 2014 \\
\hline
\end{tabular}


A
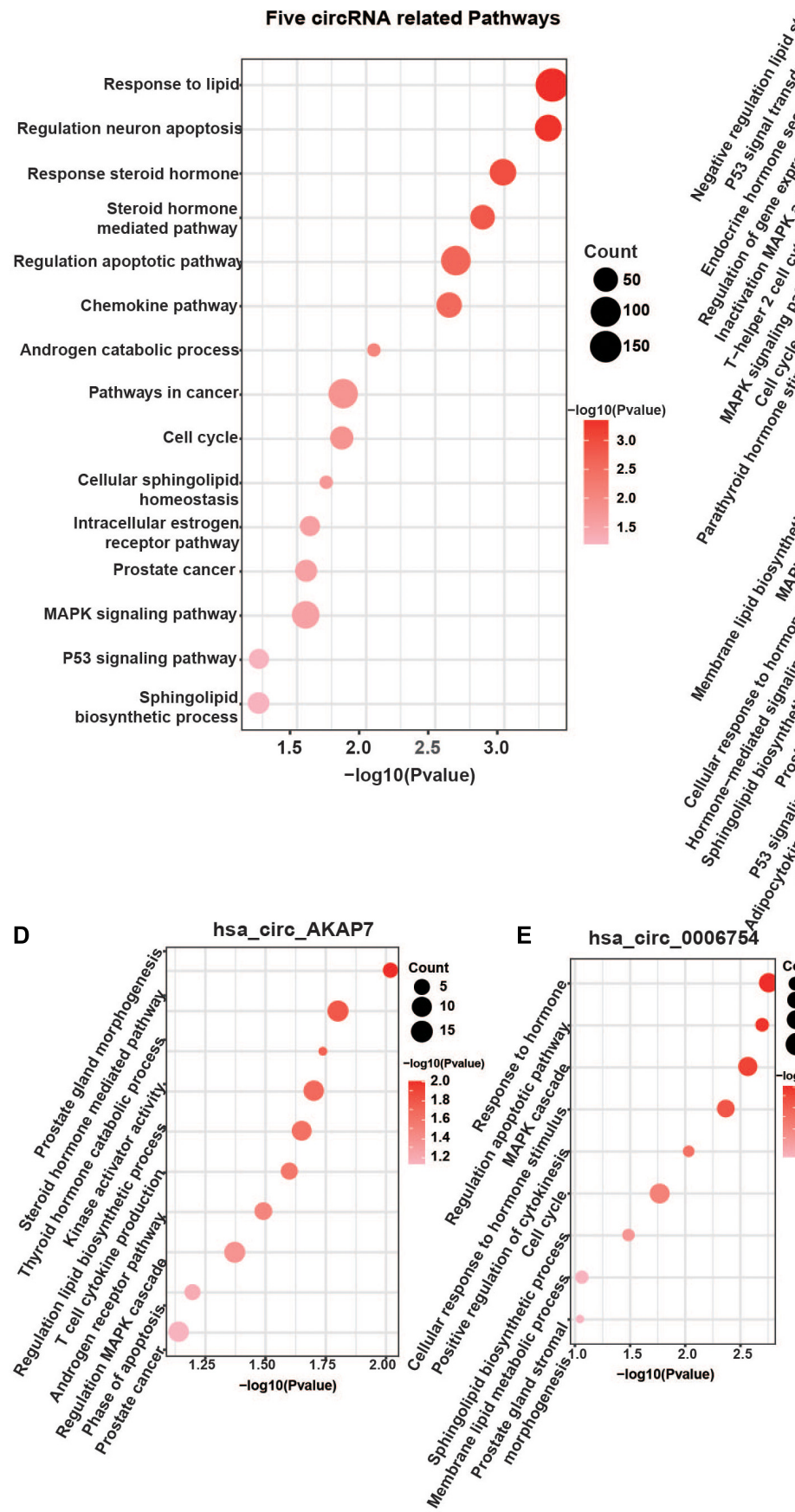

B hsa_circ_0006410
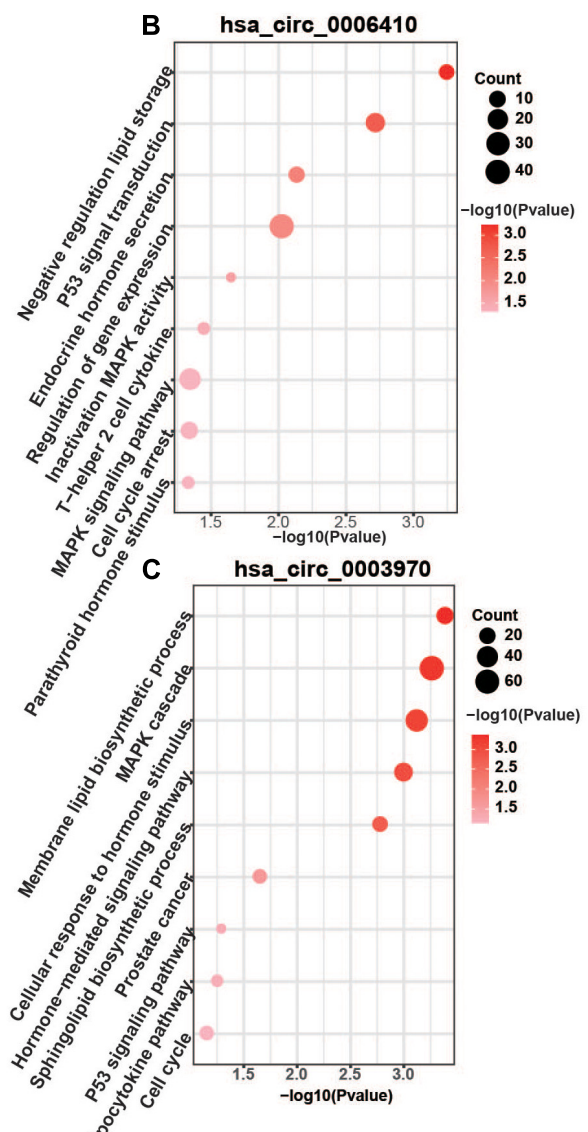

$F$

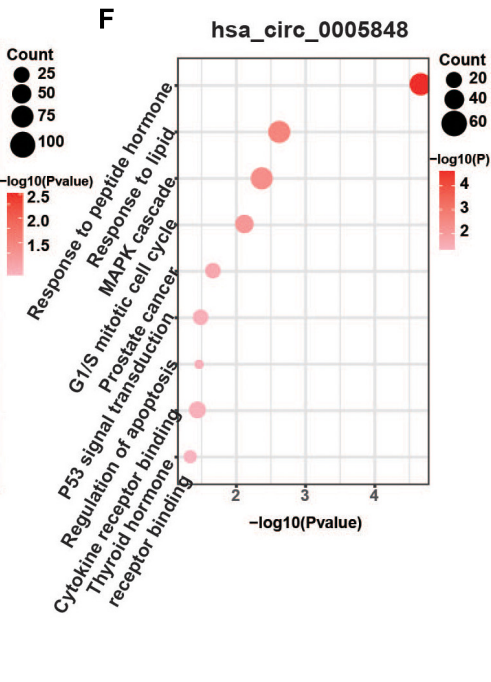

FIGURE 4 | Five specific circRNA-related pathways. (A) The five circRNA-related pathways. Only differentially expressed mRNAs with prediction score higher than 90 were considered. Well-known PCa-related pathways, such as the MAPK signaling pathway, P53 pathway, AR pathway, cell cycle, steroid hormone-mediated signaling pathway, and lipid-related process, were all found. (B) hsa_circ_0006410 related pathways. (C) hsa_circ_0003970 related pathways. (D) hsa_circ_AKAP7 related pathways. (E) hsa_circ_0006754 related pathways. (F) hsa_circ_0005848 related pathways. "Count" represents the number of genes in the relevant categories. 


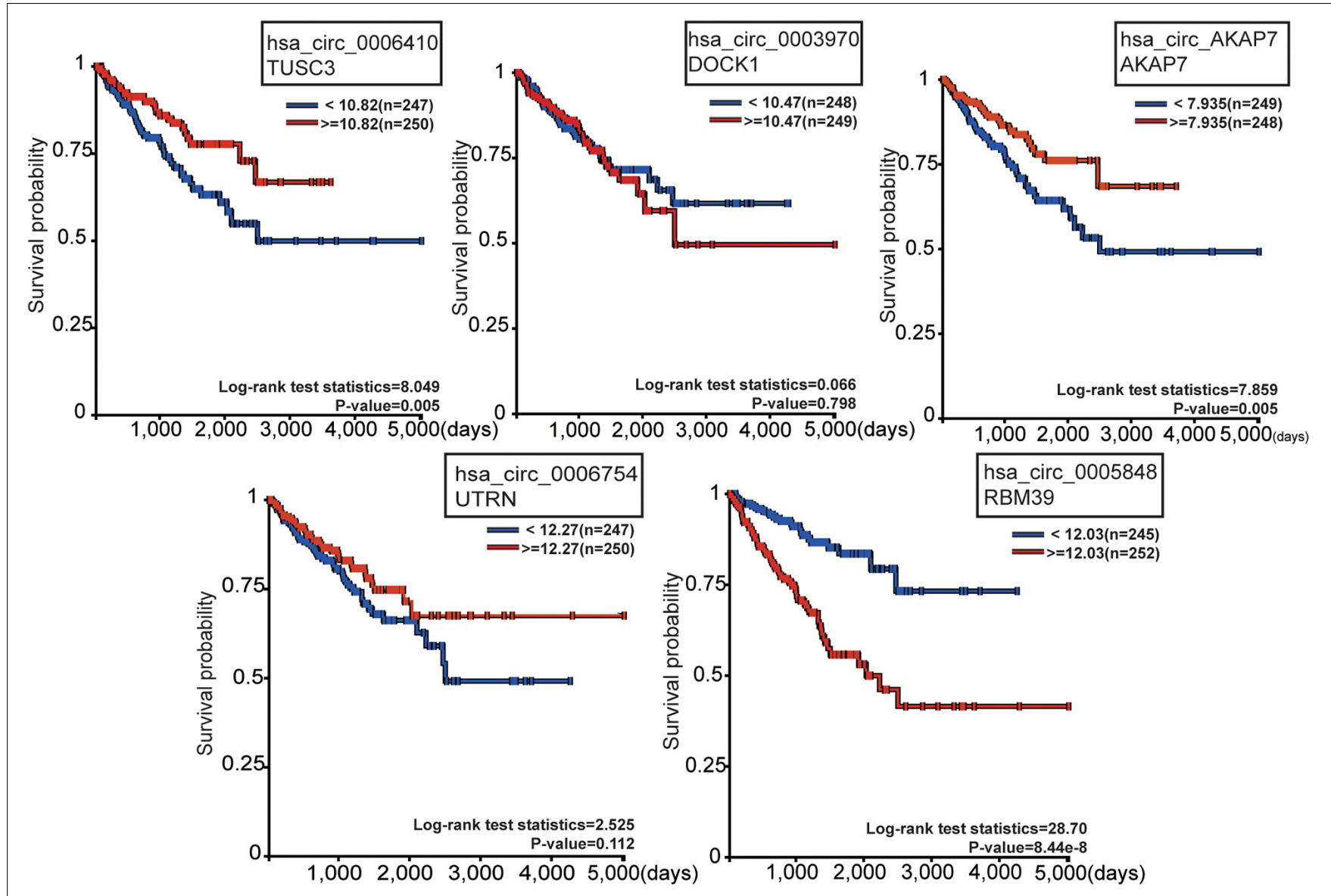

FIGURE $\mathbf{5}$ | Survival analysis of the five circRNA cognate genes in PCa. Among these, TUSC3, AKAP7, and RBM39 were significantly related with survival probability. The red line represents high gene expression, and the blue line represents low gene expression.

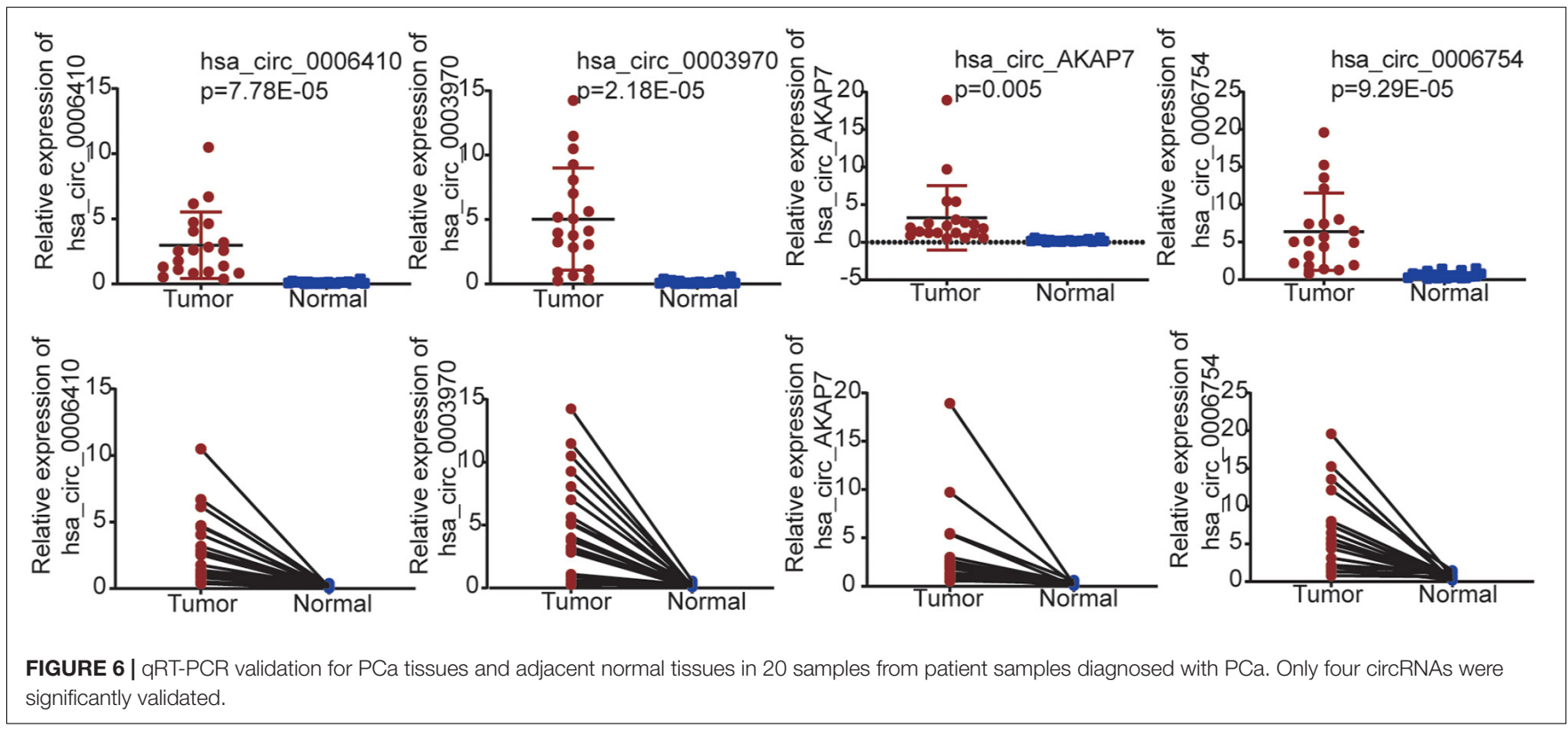


pathway, apoptosis and transcriptional misregulation in cancer (Figure 4A).

\section{Survival Analysis of CircRNA Cognate Genes and qRT-PCR Validation of Five CircRNAs}

To observe the effect of the five circRNAs in PCa patients, survival analysis of circRNA cognate genes in TCGA data was performed (Figure 5). TUSC3 (hsa_circ_0006410 cognate gene), AKAP7 (hsa_circ_AKAP7 cognate gene), and RBM39 (hsa_circ_0005848 cognate gene) were all significantly associated with progressionfree survival of PCa patients, as shown by the Kaplan-Meier plot $(P$-value $<0.05)$ (Figure 5A). Patients with high expression of TUSC3 and AKAP7 showed better overall survival. This is consistent with the fact that TUSC3 is a tumor suppressor gene (Yu et al., 2017). Meanwhile, low expression of RBM39 was found to be associated with low overall survival. RBM39 is associated with precursor messenger RNA (pre-mRNA) splicing factors, and inactivation of RBM39 causes aberrant pre-mRNA splicing. Previous studies have shown that several single amino acid substitutions in RBM39 confer resistance to the toxic effects of indisulam in cultured cancer cells and in mice with tumor xenografts (Han et al., 2017). Since the direction of differential expression varied among the five mRNAs, we think that circRNAs might act as oncogenes or tumor suppressor genes in PCa. The direction of different functional circRNAs is different in its cognate mRNAs (Figure 5). However, further experiments are required for confirming this.

We used qRT-PCR to validate the five circRNAs in $20 \mathrm{PCa}$ and normal samples (Figure 6). In our results, four circRNAs were significantly validated-hsa_circ_0006410, hsa_circ_0003970, hsa_circ_AKAP7, and hsa_circ_0006754. The relative expression of circRNAs indicated that these circRNAs were highly expressed in tumor tissues compared to normal tissues and validated our analysis.

\section{DISCUSSION}

Based on sequencing data of PCa tissues and adjacent normal tissues, we identified differentially expressed circRNAs in PCa. We filtered five specific highly expressed circRNAs that had never been studied in PCa before for further analysis. We found that the miRNAs and mRNA pathways related to these circRNAs were related to known metabolic pathways, such as PI3K-Akt signaling pathway, MAPK signaling pathway, and lipid metabolic process. This also confirmed the reliability of our findings. Through bioinformatics analysis, we analyzed expression levels of circRNA through linear RNA expression level adjustment using CIRCexplorer3. For these five circRNAs, the expression of circRNAs and mRNAs in tumor tissue was highly correlated, which is consistent with the results of previous studies. However, the fold changes of circRNAs expression were notably larger than those of their cognate mRNAs, suggesting that circRNAs play a role in tumor tissues. The underlying mechanism, however, is still unknown and requires further research.
We predicted circRNAs function by using circRNA-miRNAmRNA interactions and showed that they were all significantly enriched in the lipid metabolism pathway. The link between PCa development and lipid metabolism is well established, with AR intimately involved in a number of lipogenic processes. Altered lipid signatures may offer insights into metabolic reprogramming. Lipid pathway deregulation in advanced PCa is a hot research field to identify a therapeutic pathway. Several therapeutic agents, such as warfarin, atostatin, and orlistat, are known to block key processes in lipid metabolism and negatively influence PCa progression. Lipid metabolism is also activated by the PI3K-Akt signaling pathway by sterol regulatory element-binding protein 1 (SREBP1) (Edlind and Hsieh, 2014). The hsa_circ_0006410, hsa_circ_0003970, hsa_circ_0006754, hsa_circ_0005848, and hsa_circ_AKAP7 were all enriched in lipid related pathways, which shows their potential as targets.

The PI3K-Akt signaling pathway is deregulated in $42 \%$ of localized disease and 100\% of advanced-stage disease in PCa. This implies that the alteration of this pathway is another factor in the development of CRPC. Gene amplifications, mutations, and changes in mRNA expression of PI3K signaling pathway are highly correlated with PCa patients (Edlind and Hsieh, 2014). Hsa_circ_0006410, hsa_circ_0003970, hsa_circ_0005848, and hsa_circ_AKAP7 were all related with this pathway. CircRNAs have been reported to activate the PI3K/Akt signaling pathway by regulating gene expression in $\mathrm{PCa}$ (Wang et al., 2020). Although the exact mechanism affecting PI3K/Akt signaling pathway is unclear, the circRNAs identified in this study also provided support for this field.

We used survival analysis and qRT-PCR to validate our findings. Survival analysis is a good indicator to assess the function of genes. Three cognate genes of these five circRNAs were significantly identified in survival analysis, alluding that their cognate genes were key genes in regulating tumor progression. Meanwhile, four circRNAs were well verified by qRT-PCR, except for hsa_circ_0005848. We inferred that this may be due to the space structure or the false positive expression of hsa_circ_0005848.

Our research was based on the bioinformatics analysis of RNA-seq between prostate tumor tissues and adjacent normal tissues. We found five specific circRNAs that were highly related to the AR signaling pathway, MAPK signaling pathway, hormone-mediated signaling pathway, and cellular lipid metabolic process. Furthermore, survival analysis and qRTPCR validation also verified that the circRNAs were closely related to tumor progression of PCa. These five circRNAs can provide new solutions for research in this field.

\section{DATA AVAILABILITY STATEMENT}

The datasets presented in this study can be found in online repositories. The names of the repository/repositories and accession number(s) can be found below: Genome Sequence Archive for Human (https://bigd.big.ac.cn/bioproject/browse/ PRJCA003890) (accession: PRJCA003890). 


\section{ETHICS STATEMENT}

The studies involving human participants were reviewed and approved by Beijing Hospital Ethics Committee. The patients/participants provided their written informed consent to participate in this study. Written informed consent was obtained from the individual(s) for the publication of any potentially identifiable images or data included in this article.

\section{AUTHOR CONTRIBUTIONS}

LZ and WZ conceived the study and wrote the manuscript. LZ, FS, and YZ designed the detail analysis pipeline. LZ and FS did the bioinformatics analysis. WZ, HL, XT, and SX performed the experiments. MW and LW participated in revising the manuscript. All authors read and approved the final manuscript.

\section{REFERENCES}

Alessandro, M. S., Golombek, D. A., and Chiesa, J. J. (2019). Protein kinases in the photic signaling of the mammalian circadian clock. Yale J. Biol. Med. 92, 241-250.

Andrews, S. (2010). Fastqc: A Quality Control Tool for High Throughput Sequence Data. Available online at: https://www.bioinformatics.babraham.ac. uk/projects/fastqc/ (accessed August 10, 2020).

Arnaiz, E., Sole, C., Manterola, L., Iparraguirre, L., Otaegui, D., and Lawrie, C. H. (2019). CircRNAs and cancer: biomarkers and master regulators. Semin. Cancer Biol. 58, 90-99. doi: 10.1016/j.semcancer.2018.12.002

Bolger, A. M., Lohse, M., and Usadel, B. (2014). Trimmomatic: a flexible trimmer for illumina sequence data. Bioinformatics 30, 2114-2120. doi: 10.1093/ bioinformatics/btu170

Carroll, P. H., and Mohler, J. L. (2018). NCCN guidelines updates: prostate cancer and prostate cancer early detection. J. Natl. Compr. Canc. Netw. 16, 620-623. doi: 10.6004/jnccn.2018.0036

Chen, Y., and Wang, X. (2020). miRDB: an online database for prediction of functional microRNA targets. Nucleic Acids Res. 48, D127-D131. doi: 10.1093/ nar/gkz757

Deng, Z., Li, X., Wang, H., Geng, Y., Cai, Y., Tang, Y., et al. (2020). Dysregulation of circRNA_0001946 contributes to the proliferation and metastasis of colorectal cancer cells by targeting microRNA-135a-5p. Front. Genet. 11:357. doi: 10.3389/ fgene.2020.00357

Dhillon, A. S., Hagan, S., Rath, O., and Kolch, W. (2007). MAP kinase signalling pathways in cancer. Oncogene 26, 3279-3290. doi: 10.1038/sj.onc.1210421

Dituri, F., Mazzocca, A., Giannelli, G., and Antonaci, S. (2011). PI3K functions in cancer progression, anticancer immunity and immune evasion by tumors. Clin. Dev. Immunol. 2011:947858. doi: 10.1155/2011/947858

Dobin, A., Davis, C. A., Schlesinger, F., Drenkow, J., Zaleski, C., Jha, S., et al. (2013). STAR: ultrafast universal RNA-seq aligner. Bioinformatics 29, 15-21. doi: 10.1093/bioinformatics/bts635

Edlind, M. P., and Hsieh, A. C. (2014). PI3K-AKT-mTOR signaling in prostate cancer progression and androgen deprivation therapy resistance. Asian J. Androl. 16, 378-386. doi: 10.4103/1008-682X.122876

Eidelman, E., Twum-Ampofo, J., Ansari, J., and Siddiqui, M. M. (2017). The metabolic phenotype of prostate cancer. Front. Oncol. 7:131. doi: 10.3389/fonc. 2017.00131

Etzioni, R., and Nyame, Y. A. (2020). Prostate cancer screening guidelines for Black men: spotlight on an empty stage. J. Natl. Cancer Inst. djaa172. doi: 10.1093/jnci/djaa172

Feng, Y., Yang, Y., Zhao, X., Fan, Y., Zhou, L., Rong, J., et al. (2019). Circular RNA circ0005276 promotes the proliferation and migration of prostate cancer

\section{FUNDING}

This work was supported by National Natural Science Foundation of China (Grant 81902618), CAMS Innovation Fund for Medical Sciences (2018-I2M-1-002), Beijing Hospital Nova Project (No. BJ-2020-083), and Beijing Hospital Project (No. BJ-2019-153). Beijing Dongcheng District Outstanding Talent Funding Project (BJ-2020-047 and 2019WJGW-10-01).

\section{ACKNOWLEDGMENTS}

The authors would like to thank National Genomics Data Center, China National Center for Bioinformation/Beijing Institute of Genomics, Chinese Academy of Sciences for the data transmission. We would like to thank editage (www.editage.cn) for English language editing.

cells by interacting with FUS to transcriptionally activate XIAP. Cell Death Dis. 10:792. doi: 10.1038/s41419-019-2028-9

Goldman, M. J., Craft, B., Hastie, M., Repecka, K., McDade, F., Kamath, A., et al. (2020). Visualizing and interpreting cancer genomics data via the Xena platform. Nat. Biotechnol. 38, 675-678. doi: 10.1038/s41587-020-0546-8

Han, T., Goralski, M., Gaskill, N., Capota, E., Kim, J., Ting, T. C., et al. (2017). Anticancer sulfonamides target splicing by inducing RBM39 degradation via recruitment to DCAF15. Science 356:eaal3755. doi: 10.1126/science.aal3755

Hansen, T. B., Jensen, T. I., Clausen, B. H., Bramsen, J. B., Finsen, B., Damgaard, C. K., et al. (2013). Natural RNA circles function as efficient microRNA sponges. Nature 495, 384-388. doi: 10.1038/nature11993

Jeck, W. R., and Sharpless, N. E. (2014). Detecting and characterizing circular RNAs. Nat. Biotechnol. 32, 453-461. doi: 10.1038/nbt.2890

Jeck, W. R., Sorrentino, J. A., Wang, K., Slevin, M. K., Burd, C. E., Liu, J., et al. (2013). Circular RNAs are abundant, conserved, and associated with ALU repeats. RNA 19, 141-157. doi: 10.1261/rna.035667.112

Kitagawa, Y., Ueno, S., Izumi, K., Kadono, Y., Konaka, H., Mizokami, A., et al. (2014). Cumulative probability of prostate cancer detection in biopsy according to free/total PSA ratio in men with total PSA levels of $2.1-10.0 \mathrm{ng} / \mathrm{ml}$ at population screening. J. Cancer Res. Clin. Oncol. 140, 53-59. doi: 10.1007/ s00432-013-1543-9

Lander, E. S., Linton, L. M., Birren, B., Nusbaum, C., Zody, M. C., Baldwin, J., et al. (2001). Initial sequencing and analysis of the human genome. Nature 409, 860-921. doi: $10.1038 / 35057062$

Li, S., Yu, C., Zhang, Y., Liu, J., Jia, Y., Sun, F., et al. (2020). Circular RNA cir-ITCH is a potential therapeutic target for the treatment of castration-resistant prostate cancer. Biomed. Res. Int. 2020:7586521. doi: 10.1155/2020/7586521

Li, Y., Xie, J., Xu, X., Wang, J., Ao, F., Wan, Y., et al. (2013). MicroRNA-548 downregulates host antiviral response via direct targeting of IFN-lambda1. Protein Cell 4, 130-141. doi: 10.1007/s13238-012-2081-y

Liao, Y., Smyth, G. K., and Shi, W. (2014). featureCounts: an efficient general purpose program for assigning sequence reads to genomic features. Bioinformatics 30, 923-930. doi: 10.1093/bioinformatics/btt656

Lin, P., Zhu, L., Sun, W., Yang, Z., Sun, H., Li, D., et al. (2018). Prostate cancer cell proliferation is suppressed by microRNA-3160-5p via targeting of F-box and WD repeat domain containing 8. Oncol. Lett. 15, 9436-9442. doi: 10.3892/ol. 2018.8505

Lin, Y. C., Lin, J. F., Tsai, T. F., Chou, K. Y., Chen, H. E., and Hwang, T. I. (2017). Tumor suppressor miRNA-204-5p promotes apoptosis by targeting BCL2 in prostate cancer cells. Asian J. Surg. 40, 396-406. doi: 10.1016/j.asjsur.2016.07.001

Litwin, M. S., and Tan, H. J. (2017). The diagnosis and treatment of prostate cancer: a review. JAMA 317, 2532-2542. doi: 10.1001/jama.2017.7248 
Liu, M., Wang, Q., Shen, J., Yang, B. B., and Ding, X. (2019). Circbank: a comprehensive database for circRNA with standard nomenclature. RNA Biol. 16, 899-905. doi: 10.1080/15476286.2019.1600395

Ma, X. K., Wang, M. R., Liu, C. X., Dong, R., Carmichael, G. G., Chen, L. L., et al. (2019). CIRCexplorer3: a Clear pipeline for direct comparison of circular and linear RNA expression. Genom. Proteom. Bioinform. 17, 511-521. doi: 10.1016/ j.gpb.2019.11.004

Martinez, F. O., Helming, L., and Gordon, S. (2009). Alternative activation of macrophages: an immunologic functional perspective. Annu. Rev. Immunol. 27, 451-483. doi: 10.1146/annurev.immunol.021908.132532

Mekhail, S. M., Yousef, P. G., Jackinsky, S. W., Pasic, M., and Yousef, G. M. (2014). miRNA in prostate cancer: new prospects for old challenges. EJIFCC 25, 79-98.

Munkley, J., Vodak, D., Livermore, K. E., James, K., Wilson, B. T., Knight, B., et al. (2016). Glycosylation is an androgen-regulated process essential for prostate cancer cell viability. EBioMedicine 8, 103-116. doi: 10.1016/j.ebiom.2016.04.018

PCa (2019). NICE guidance - prostate cancer: diagnosis and management: (c) NICE (2019) Prostate cancer: diagnosis and management. BJU Int. 124, 9-26. doi: 10.1111/bju.14809

Qin, X., Zhang, J., Lin, Y., Sun, X. M., Zhang, J. N., and Cheng, Z. Q. (2020). Identification of MiR-211-5p as a tumor suppressor by targeting ACSL4 in Hepatocellular carcinoma. J. Transl. Med. 18:326. doi: 10.1186/s12967-02002494-7

Robinson, M. D., McCarthy, D. J., and Smyth, G. K. (2010). edgeR: a Bioconductor package for differential expression analysis of digital gene expression data Bioinformatics 26, 139-140. doi: 10.1093/bioinformatics/btp616

Salzman, J., Gawad, C., Wang, P. L., Lacayo, N., and Brown, P. O. (2012). Circular RNAs are the predominant transcript isoform from hundreds of human genes in diverse cell types. PLoS One 7:e30733. doi: 10.1371/journal.pone.0030733

Shannon, P., Markiel, A., Ozier, O., Baliga, N. S., Wang, J. T., Ramage, D., et al. (2003). Cytoscape: a software environment for integrated models of biomolecular interaction networks. Genome Res. 13, 2498-2504. doi: 10.1101/ gr.1239303

Shi, Y., Qiu, M., Wu, Y., and Hai, L. (2015). MiR-548-3p functions as an antioncogenic regulator in breast cancer. Biomed. Pharmacother. 75, 111-116. doi: 10.1016/j.biopha.2015.07.027

Siegel, R. L., Miller, K. D., and Jemal, A. (2017). Cancer statistics, 2017. CA Cancer J. Clin. 67, 7-30. doi: 10.3322/caac.21387

Sun, Y., Hu, B., Wang, Y., Li, Z., Wu, J., Yang, Y., et al. (2018). miR-216a-5p inhibits malignant progression in small cell lung cancer: involvement of the Bcl-2 family proteins. Cancer Manag. Res. 10, 4735-4745. doi: 10.2147/CMAR.S178380

Suzuki, H., Zuo, Y., Wang, J., Zhang, M. Q., Malhotra, A., and Mayeda, A. (2006). Characterization of RNase R-digested cellular RNA source that consists of lariat and circular RNAs from pre-mRNA splicing. Nucleic Acids Res. 34:e63. doi: $10.1093 / \mathrm{nar} / \mathrm{gkl151}$
Tamura, K., Makino, A., Hullin-Matsuda, F., Kobayashi, T., Furihata, M., Chung, S., et al. (2009). Novel lipogenic enzyme ELOVL7 is involved in prostate cancer growth through saturated long-chain fatty acid metabolism. Cancer Res. 69, 8133-8140. doi: 10.1158/0008-5472.CAN-09-0775

Tay, Y., Rinn, J., and Pandolfi, P. P. (2014). The multilayered complexity of ceRNA crosstalk and competition. Nature 505, 344-352. doi: 10.1038/nature12986

Wang, Y., Yin, L., and Sun, X. (2020). CircRNA hsa_circ_0002577 accelerates endometrial cancer progression through activating IGF1R/PI3K/Akt pathway. J. Exp. Clin. Cancer Res. 39:169. doi: 10.1186/s13046-020-01679-8

Wei, S., Zhang, Z. Y., Fu, S. L., Xie, J. G., Liu, X. S., Xu, Y. J., et al. (2016). Hsa-miR623 suppresses tumor progression in human lung adenocarcinoma. Cell Death Dis. 7:e2388. doi: 10.1038/cddis.2016.260

Wickham, H. (2016). ggplot2: Elegant Graphics for Data Analysis. New York, NY: Springer-Verlag.

Wu, X., Daniels, G., Lee, P., and Monaco, M. E. (2014). Lipid metabolism in prostate cancer. Am. J. Clin. Exp. Urol. 2, 111-120.

Yang, Q., Wu, J., Zhao, J., Xu, T., Zhao, Z., Song, X., et al. (2018). Circular RNA expression profiles during the differentiation of mouse neural stem cells. BMC Syst. Biol. 12(Suppl. 8):128. doi: 10.1186/s12918-0180651-1

Young, M. D., Wakefield, M. J., Smyth, G. K., and Oshlack, A. (2010). Gene ontology analysis for RNA-seq: accounting for selection bias. Genome Biol. 11:R14. doi: 10.1186/gb-2010-11-2-r14

Yu, T., Wang, Y., Fan, Y., Fang, N., Wang, T., Xu, T., et al. (2019). CircRNAs in cancer metabolism: a review. J. Hematol. Oncol. 12:90. doi: 10.1186/s13045019-0776-8

Yu, X., Zhai, C., Fan, Y., Zhang, J., Liang, N., Liu, F., et al. (2017). TUSC3: a novel tumour suppressor gene and its functional implications. J. Cell. Mol. Med. 21, 1711-1718. doi: 10.1111/jcmm. 13128

Zhang, X. O., Dong, R., Zhang, Y., Zhang, J. L., Luo, Z., Zhang, J., et al. (2016). Diverse alternative back-splicing and alternative splicing landscape of circular RNAs. Genome Res. 26, 1277-1287. doi: 10.1101/gr.2028 95.115

Conflict of Interest: The authors declare that the research was conducted in the absence of any commercial or financial relationships that could be construed as a potential conflict of interest.

Copyright (c) 2021 Zhang, Zhang, Li, Tang, Xu, Wu, Wan, Su and Zhang. This is an open-access article distributed under the terms of the Creative Commons Attribution License (CC BY). The use, distribution or reproduction in other forums is permitted, provided the original author(s) and the copyright owner(s) are credited and that the original publication in this journal is cited, in accordance with accepted academic practice. No use, distribution or reproduction is permitted which does not comply with these terms. 